\title{
Correlation of Nutritional Status and Pressure Ulcers Healing in Spinal Cord Injury Subjects : A Non-randomized, Non-controlled Quasi Experimental Study
}

\author{
Amit Kumar Bhagat ${ }^{1}$, Rajeshwar Nath Srivastava ${ }^{2 *}$, Mukesh kumar Dwivedi ${ }^{2}$, \\ Sudeepti Ratan Srivastava ${ }^{2}$, Saloni Raj ${ }^{3}$ and Abhijit Chandra ${ }^{1}$ \\ ${ }^{1}$ Dept. of Surgical Gastroenterology, King George's Medical University, Lucknow, U.P., India, \\ ${ }^{2}$ Dept. of Orthopaedic Surgery, King George's Medical University, Lucknow, U.P., India \\ ${ }^{3}$ MBBS Intern, King George's Medical University, Lucknow, U.P., India
}

\begin{abstract}
Objective: This study was done to determine the cause effect relationship between pressure ulcers (PUs) and metabolic alterations in individual having PUs with Spinal Cord Injury (SCI)

Methods: 123 SCI subjects with PUs were enrolled in the study. PUs were measured for surface area, exudate, depth and formation of granulation tissue using the pressure ulcers scale for healing tool. The nutritional status of subjects was assessed by measuring serum haemoglobin, albumin and transferrin levels at baseline (week 0), weeks 3 and 6 during follow-up.

Results: An inverse correlation of wound size was observed with nutritional status at weeks 3 and 6 . There was a significant increase ( $p<$ $0.001)$ in: Haemoglobin $(10.7-12.4 \mathrm{~g} / \mathrm{dl})$, Albumin $(2.9$ - $4.04 \mathrm{~g} / \mathrm{dl})$ and Transferrin $(174.1-186.9 \mu \mathrm{g} / \mathrm{dl})$ levels at the time of discharge. The size of PUs was drastically reduced up to 5.4 fold at the time of discharge.

Conclusion: Given the high prevalence of malnutrition among subjects with PUs, performing a routine nutritional screening should result in early identification of residents with the risk of developing PUs. This article gives statistical evidence that healing of PUs is associated with an increase in $\mathrm{Hb}$ level and improving metabolic alterations. There seems to be a positive relationship between PU healing cascade and nutritional corrections. In future, this relation may be disclosed with newer intervention.
\end{abstract}

Keywords: Pressure Ulcer, Spinal Cord Injury, Nutritional Biomarkers

\section{Introduction}

Pressure ulcers (PUs), also known as pressure injuries, are initiated by sustained pressure on the skin. They occur as areas of necrosis when tissue gets compressed over bony prominences and more so in spinal cord injuries (SCI) where the skin is often anesthetized. ${ }^{[1]}$ Other factors contributing to PUs in SCI include immobility, constant pressure and moisture ${ }^{[2]}$ Management of PUs is an important clinical challenge to the health care systems and there is no gold standard has yet been established for their treatment.

Nutrition plays an important role in wound healing. PU is one of the wounds that in particular require good nutrition to prevent its progression and for early recovery. SCI subjects are more prone to have nutritional deficiencies due to decreased appetite resulting from loss of movement and bladder- bowel control. ${ }^{[3]}$ Several studies have suggested that subjects with poor nutritional status are more prone for prolonged hospital stay, occurrence of PUs and high mortality in comparison to those who are well nourished. ${ }^{[4]}$ Protein deficiency slows the fibroblastic response and formation of new blood vessels to the PU site and play an important role in delayed healing. ${ }^{[5]}$ Hypoalbuminemia has been shown to increase mortality in different clinical settings while decreased $\mathrm{Hb}$ level was significantly associated with PU formation in traumatic brain injury ${ }^{[6]}$

Although nutritional status and wound healing have strong correlations, relationship between the two is often debated. The human liver is classically perceived as a non-immunological organ, required for metabolic activities, nutrient storage and detoxification. ${ }^{[7]}$ Liver is also an immunologically complex organ, responsible for the production of acute phase proteins, complement components, cytokines and chemokines, and contains large, diverse populations of resident immune cells. ${ }^{[8,9,10]}$ Even in the healthy liver, constantly changing metabolic and tissue remodeling activity, when gets combined with regular exposure to microbial products, results in persistent, regulated inflammation. Inflammation is essential to combat infection and in conditions that persist in PUs, chronic inflammation ensues. ${ }^{[7]}$ This is 
an immune response that persists for months, in which inflammation, tissue remodeling and repair processes occur simultaneously. It can be induced by a number of different insults; however, regardless of etiology, chronic inflammation induces fibrosis that eventually leads to hepatic dysfunction which inhibits the use of iron stored in the reticuloendothelial system ${ }^{[11,12]}$ and the hepatic synthesis of albumin ${ }^{[13,14,15,16]}$ It is still not certain whether the metabolic alterations seen in PUs are the result of hepatic dysfunction in chronic inflammatory status or because of nutritional deficiencies through diet. ${ }^{[17]}$

This study was done to determine the cause effect relationship between the PUs and the metabolic alterations in individual with SCI having PUs. We hypothesized that a rigorous, meticulous and zealous care of the wound is essential to correct the metabolic alterations being precipitated by liver dysfunction due to chronic inflammatory status seen in PUs of SCI.

\section{Methodology}

Study Design, Setting and Period: This study was conducted in the SCI unit of Orthopedic Surgery Department from Oct. 2012 to Sept. 2015. A total of 123 SCI subjects who were admitted in SCI unit and fulfilled the inclusion criteria were enrolled in the study. At baseline a complete medical history, socio-demographic data, clinical assessment of wounds and nutritional assessment of subjects were recorded. Clinical photography of PUs was done for healing assessment at each follow-up. The study was approved by the institutional ethics committee (IEC) of the university (KGMU). A Written informed consent was obtained either from the subjects or their caregivers.

Inclusion and Exclusion Criteria: Individuals with SCI, aged between 16 to 60 years having PUs of stage I to IV were enrolled in the study. Stages of PUs were defined by the NPUAP taxonomy. Exclusion criteria were PUs with necrotic tissue that could not be removed on baseline debridement, chronic osteomyelitis, exposed blood vessels and specific co-morbid conditions likely to impair wound healing such as diabetes mellitus, rheumatoid disease and vasculitis.

Clinical Assessment of PUs: All clinical assessments were done by the same investigator throughout the study. Using the pressure ulcers scale for healing (PUSH) tool, version 30 (Copyright 2003, NPUAP), each PU was measured for its greatest length and width with a measuring device enabling measurements in centimeters. The volume of exudate produced by the wound was categorized as none (0), light (1), moderate (2), or heavy (3) after dressing removal. In addition, the PUSH tool was used to evaluate the presence of necrotic tissue, slough and red-granulation tissue. The depth of PUs was also measured with a cotton tip applicator, which was inserted into the ulcers and marked at the deepest level at every follow-up. ${ }^{[18]}$

\begin{abstract}
Algorithm of PU care - Debridement, dressing and offloading: Standardized wound care of PUs was done under aseptic conditions in all the cases. Surgical debridement with 23 no. blades was done in PUs of grade III and IV at the time of enrollment. All PUs were irrigated by normal saline and cleansing agents (hydrogen peroxide and chlorine water) for chemical debridement and removal of slough at every change of dressing. Following irrigation, proteolytic enzymes and antimicrobial cream (Metrogyl Povidone ointment) was used to cover the raw surface of the PU. Wet-to-dry gauze dressing was used to cover the wound and to protect the wound and neighboring skin from microbial and physical insult. The dressing was changed either twice a day or once daily depending on the amount of exudates.
\end{abstract}

Off- loading of the PU area was done every 2 hours for 15-20 minutes to release the pressure on PU either by subjects or their attendants (when subjects found it difficult to perform a weight shift maneuver). Air cushions were sometimes used to help achieve the necessary off-loading of pressure to facilitate PU healing. These cushions were custom designed for redistribution of pressure at another area.

Assessment of Nutritional Status: Serum Hb, serum albumin and serum transferrin levels were measured at baseline (week 0), weeks 3 and 6 during follow-up. A selfstandardized oral nutritional supplement was provided to all subjects. The recommended dose of NS was one capsule twice a day. The two capsules provided $250 \mathrm{kcal} / 20 \mathrm{~g}$ protein, $3 \mathrm{~g}$ arginine, $500 \mathrm{mg}$ elemental calcium, $50 \mathrm{mg}$ Magnesium oxide, $250 \mathrm{mg}$ Vitamin C, 38mg Vitamin E, 35mg Zinc, 100mg L-lysine, 500IU Cholecalciferol, $750 \mathrm{mcg}$ Methylcobalamine, $10 \mathrm{mg}$ beta carotene and other micronutrients like folic acid, selenium and lycopene. Whereas NS was provided to improve the general health of the subjects, no attempt was made to correct the nutritional deficiencies observed in the three nutritional markers studied at baseline.

Statistical Analysis: Statistical analysis was carried out using software SPSS version 16.0 (Chicago, Inc., USA). The results were presented as mean $\pm \mathrm{SD}$ or frequency and percentage as appropriate. Paired t-test was used to compare the continuous parameters at baseline and at the follow-ups. The p-value $<0.05$ was considered significant. 


\section{Results}

Total 123 SCI subjects were participating in the study. Out of these, 68 were paraplegic and 55 were quadriplegic. The age of participants ranged between 13 - 59 years with a mean age of $32.27 \pm 9.138$ years. (Table 1) The sex ratio in the study population showed that male patient proportion was much higher than female: $91.9 \%$ and $8.1 \%$ respectively. Most of the subjects were from Grade-II (36.6\%) and Grade-III (30.9\%) type of Pressure Ulcer (PU); $20 \%$ subjects had Grade-IV type and very few subjects (10\%) had Grade-I type of PUs. Out of 123 subjects, 53 subjects $(43.1 \%)$ had light exudates; 31 subjects $(25.2 \%)$ had moderate exudate and 10 subjects $(8.1 \%)$ had heavy exudates from PU. Among all SCI cases enrolled, ASIA Score A and B subjects were more prevalent (64:19\%). It was found that 79 subjects $(64.23 \%)$ developed SCI due to fall from height; 24 subjects (19.5\%) developed SCI after a motor vehicle accident and 8 subjects $(6.5 \%)$ developed SCI due to carrying heavy weight.
Correlation of Pressure Ulcers with other Parameters: Subjects were observed with elevated wound size in higher grade of pressure ulcer at the time of admission (0 week) (Figure 1). The nutritional status (at $3^{\text {rd }}$ and $6^{\text {th }}$ week) of the admitted patients was elevated and a reduction in wound size of pressure ulcer was observed (Table 2). An inverse correlation of wound size was observed with nutritional status at weeks 3 and 6 . There was a significant increase ( $p$ $<0.001)$ in: Haemoglobin (10.7 - $12.4 \mathrm{~g} / \mathrm{dl})$, Albumin (2.9 $4.04 \mathrm{~g} / \mathrm{dl})$ and Transferrin $(174.1-186.9 \mu \mathrm{g} / \mathrm{dl})$ levels at the time of discharge. The univariate analysis of ulcer size with various nutritional parameters was done at follow-up weeks of the study. A significant negative correlation of ulcer size was observed with theses parameters at weeks 3 and 6 . The size of PUs was drastically reduced up to 5.4 fold at the time of discharge (Table 3 ). The amount of exudates were reduced during follow-up from week 0 to $6^{\text {th }}$ week (Light, $2.20 \pm 0.18$ to $0.82 \pm 0.007$; Moderate, $3.15 \pm 0.2$ to $2.16 \pm$ 0.358 ; Heavy, $4.50 \pm 0.4$ to $3.1 \pm 0.404$ ). (Table 4)

Table 1: Characteristics of study population.

\begin{tabular}{|c|c|c|}
\hline Variables & No. of patients & Percent (\%) \\
\hline \multicolumn{3}{|c|}{ Sex (n) (frequency) } \\
\hline Male & 113 & 91.9 \\
\hline Female & 10 & 8.1 \\
\hline Total & 123 & 100 \\
\hline \multicolumn{3}{|c|}{ Exudates } \\
\hline None & 29 & 23.6 \\
\hline Light & 53 & 43.1 \\
\hline Moderate & 31 & 25.2 \\
\hline Heavy & 10 & 8.1 \\
\hline \multicolumn{3}{|c|}{ Grade } \\
\hline Grade I & 13 & 10.6 \\
\hline Grade II & 45 & 36.6 \\
\hline Grade III & 38 & 30.9 \\
\hline Grade IV & 27 & 22.0 \\
\hline \multicolumn{3}{|c|}{ SCI Level } \\
\hline Quadriplegia & 55 & 44.71 \\
\hline Paraplegia & 68 & 55.28 \\
\hline \multicolumn{3}{|c|}{ ASIA Score } \\
\hline A & 79 & 64.23 \\
\hline$B$ & 24 & 19.51 \\
\hline $\mathrm{C}$ & 11 & 8.94 \\
\hline$D$ & 9 & 7.31 \\
\hline
\end{tabular}


Variables

No. of patients

Percent (\%)

SCI Etiology

\begin{tabular}{|c|c|c|}
\hline Fall from Height & 79 & 64.23 \\
\hline Fall of Weight & 08 & 6.5 \\
\hline Motor Vehicle Crash & 24 & 19.5 \\
\hline Miscellaneous & 12 & 9.8 \\
\hline
\end{tabular}

\section{Education States:}

Less than high school 6855.28

High school/+2 3931.70

Bachelor's Degree 108.13

Master Degree 64.87

Values are presented in percentage.

Table 2: Average wound size and levels of biochemical indexes of different grades at the time of admission and follow-up.

\begin{tabular}{|c|c|c|c|c|}
\hline Grades & Average Wound Size & Average Hemoglobin & Average Albumin & Average Transferrin \\
\hline \multicolumn{5}{|c|}{ At the time of admission } \\
\hline 1 & $41.52 \pm 2.6$ & $9.8 \pm 0.5$ & $3 \pm 0.04$ & $175.7 \pm 65.1$ \\
\hline II & $42.5 \pm 1.8$ & $10.6 \pm 1.1$ & $2.8 \pm 0.02$ & $176.7 \pm 42.9$ \\
\hline III & $47.59 \pm 3.7$ & $11.09 \pm 1.3$ & $2.9 \pm 0.1$ & $175.3 \pm 49.4$ \\
\hline IV & $54.5 \pm 4.3$ & $10.6 \pm 0.98$ & $3.1 \pm 0.3$ & $170.4 \pm 68.4$ \\
\hline \multicolumn{5}{|c|}{$3^{\text {rd }}$ week after admission } \\
\hline I & $34.9 \pm 2.6$ & $10.7 \pm 1.3$ & $3.1 \pm 2.6$ & $177.3 \pm 54.1$ \\
\hline II & $39.3 \pm 2.6$ & $11 \pm 1.1$ & $3 \pm 2.6$ & $177 \pm 51.3$ \\
\hline III & $40.65 \pm 2.6$ & $11 \pm 0.8$ & $2.9 \pm 2.6$ & $177.4 \pm 76.2$ \\
\hline IV & $41.1 \pm 2.6$ & $11.2 \pm 2.3$ & $3.2 \pm 2.6$ & $174.1 \pm 67.3$ \\
\hline \multicolumn{5}{|c|}{$6^{\text {th }}$ week after admission } \\
\hline $\mathrm{I}$ & $22.3 \pm 2.6$ & $12.4 \pm 1.4$ & $3.9 \pm 0.2$ & $184.7 \pm 78.6$ \\
\hline II & $28.02 \pm 2.6$ & $12.7 \pm 1.3$ & $4.2 \pm 0.3$ & $193.2 \pm 81.3$ \\
\hline III & $29.8 \pm 2.6$ & $12.7 \pm 1.11$ & $4.1 \pm 0.3$ & $185.3 \pm 92.7$ \\
\hline IV & $34.5 \pm 2.6$ & $11.7 \pm 1.6$ & $3.8 \pm 0.4$ & $188.2 \pm 82.8$ \\
\hline
\end{tabular}

Values are presented in mean \pm standard deviation.

Table 3: Pearson correlation of various parameters with size of ulcer.

\section{Wound volume vs Number}

\begin{tabular}{|c|c|}
\hline Hb Level & 1 \\
\hline Albumin g/dl & \\
\hline Transferrin level & 1 \\
\hline
\end{tabular}

\section{Pearson ' $r$ '}

$95 \%$ confidence interval

\section{At the time of admission}

\begin{tabular}{l|l}
0.4752 & -0.8944 to 0.9860
\end{tabular}

\begin{tabular}{l|l}
0.4752 & -0.8944 to 0.9860 \\
0.6501 & -0.8290 to 0.9916
\end{tabular}

\begin{tabular}{l|l}
-0.9314 & -0.9986 to 0.2837
\end{tabular}

$3^{\text {rd }}$ week after admission

\begin{tabular}{|c|c|}
\hline Hb Level & 123 \\
\hline Albumin $\mathrm{g} / \mathrm{dL}$ & 123 \\
\hline Transferrin level & 123 \\
\hline
\end{tabular}

\begin{tabular}{|c|c|}
\hline Hb Level & 123 \\
\hline Albumin g/dl & 123 \\
\hline Transferrin level & 123 \\
\hline
\end{tabular}

\begin{tabular}{l|l|l}
\hline 123 & -0.8116 & \\
\hline 123 & -0.8208 & \\
\hline 123 & -0.7233 & -0 \\
\hline
\end{tabular}

-0.9646 to -0.2497 -0.9665 to -0.2752 -0.9459 to -0.03777

$6^{\text {th }}$ week after admission

\section{$P$ value}

\begin{tabular}{|l|l|l|l|}
\hline 23 & -0.7468 & -0.9245 to -0.3025 & $0.0053^{*}$ \\
\hline 23 & -0.7734 & -0.9331 to -0.3585 & $0.0032^{*}$ \\
\hline 23 & -0.8224 & -0.9331 to -0.3585 & $0.0010^{*}$ \\
\hline
\end{tabular}

Values are presented in percent coefficient. P value $<0.05$ is considered as significant. * Significant 
Table 4: Amount of exudates at zero week, third week and sixth week.

\begin{tabular}{|c|c|c|c|}
\hline & 0 week & $3^{\text {rd } \text { Week }}$ & 6th week \\
\hline Light & $2.20 \pm 0.18$ & $1.12 \pm 0.31$ & $0.82 \pm 0.007$ \\
\hline Moderate & $3.15 \pm 0.2$ & $2.23 \pm 0.247$ & $2.16 \pm 0.358$ \\
\hline Heavy & $4.50 \pm 0.4$ & $2.8 \pm 0.35$ & $3.1 \pm 0.404$ \\
\hline
\end{tabular}

Values are presented in mean \pm standard deviation.

Statement of Informed Consent

Statement of Human and Animal Rights

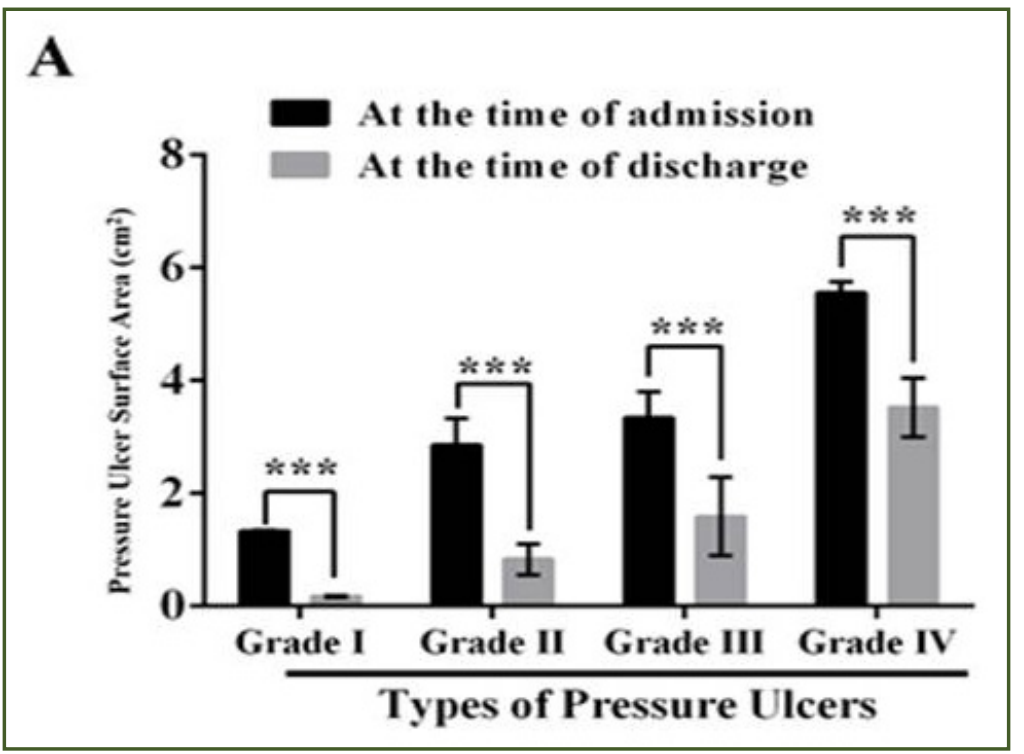

Fig. 1: Coparison of decrease in the size of uleers with different Grades of Pressure Ulcers.

\section{Discussion}

The results of the present study confirm that SCI subjects with PUs show mild to moderate anaemia and specific serum protein alterations. More importantly, the work demonstrates that the progression and healing of PUs are closely related to these metabolic alterations. PU results in hyporegenerative, normocytic, normochromic anaemia and hypoalbuminemia which is typical feature of chronic inflammatory disorder. Chronic inflammatory status inhibits the use of iron stored in the reticuloendothetial system ${ }^{[11,]}$ and the hepatic synthesis of albumin ${ }^{[12,13]}$ The disappearance of both anaemia and protein alterations soon after PU healing also supports the hypothesis that they have a close relationship; however the mechanism is not well understood.

The characteristics of anaemia and serum protein alteration and pressure ulcer area do not permit us to attribute anaemia to a loss of plasma and blood from the ulcer or to protein hypercatabolism. ${ }^{[14]}$ If protein alterations are due to a loss of plasma from the decubitus or to accelerated protein catabolism, a decrease in all protein fractions should be expected which was not found in this study. Similarly, the anaemia of SCI subjects with PU was not iron-deficiency anaemia, which is characterized by reduced Heamoglobin and increased Transferrin ${ }^{[16]}$, as in this study we found reduced $\mathrm{Hb}$ and decreased Transferrin level.

In our study we found that high to moderate amount of exudate was delaying granulation tissue formation and PU healing. This could be because of interstitial edema and loss of growth factors, collagen and fibrin in exudate which is essential for granulation tissue formation. It is known that protein-energy deficiency reduces fibroblastic cellular activity, delays angiogenesis in the proliferative stage, reduces collagen synthesis and maturation in the remodelling stage and may therefore lead to increased wound dehiscence and delayed PU healing. ${ }^{[17]}$ Also, a deficiency in serum albumin causes impaired wound perfusion and reduces the osmotic pressure in the intravascular space. This causes interstitial edema, reduces tissue oxygenation and tissue tolerance to the forces of pressure. ${ }^{[18]}$

In this study we also found that the rate of red granulation tissue formation was directly proportional to the level of $\mathrm{Hb}$. Reduced $\mathrm{Hb}$ may cause disorder in surgical and 
traumatic tissue oxygenation and embitter ischemia and inflammation that is a base for wound infection. ${ }^{[19]}$ Various studies have shown that oxygen transferred by $\mathrm{Hb}$ is involved in all steps of wound healing including inflammation, granulation, neo-angiogenesis, and tissue modeling ${ }^{[20]}$ The use of $\mathrm{Hb}$ to facilitate oxygen diffusion seems to be an ideal way to increase the oxygen supply to the wound bed and, as a result, enhance the underlying wound healing processes. One of the study supported this by observations of fast onset of proliferative processes, in many cases within the first 2-4 weeks after first application of $\mathrm{Hb}$ to the wound ${ }^{[20,21]}$ Another study suggested that anaemia and serum protein alteration tend to disappear with pressure ulcer healing. ${ }^{[22]}$ Similarly the present work demonstrates that both anaemia and serum protein alteration tend to decrease when the ulcers pass from the necrosis, inflammatory phase to the granulation phase and with the eradication of superimposed infections, establishing a close relationship between healing of ulcers and metabolic improvement.

For anaemia, subjects with pressure sores are often treated with iron therapy. ${ }^{[23]}$ Since the anaemia of these subjects is due to difficulty in using iron stores, iron treatment is not beneficial, and might even be potentially dangerous because of the possibility of iatrogenic hemochromatosis. ${ }^{[24]}$ In our study, the NS provided to the subjects did not contain any iron formulations but the $\mathrm{Hb}$ level improved with ulcer healing supporting the above hypothesis. Recently, it has been demonstrated that the administration of erythropoiesis stimulating proteins (Erythropoietin and Darbepoetin) may be effective in the treatment of this type of anemia. ${ }^{[25]}$

Our data suggests that this form of treatment should be avoided. In fact, the main cause of hypoalbuminemia is not protein loss from the ulcer but reduced hepatic synthesis secondary to the chronic inflammatory state. Furthermore, the efficacy of albumin administration to accelerate wound healing is debatable. ${ }^{[26]}$. The administration of albumin alone to treat its deficiency may not be advisable because the lack of other nutrients like essential amino acids remains. ${ }^{[27]}$ In our study the NS was rich in protein and arginine but albumin administration was not done. Protein deficiency blunts the fibroblastic response and impairs neoangiogenesis, collagen synthesis and wound remodelling. Arginine appears to effect microvascular and perfusion changes as a precursor to the synthesis of nitric oxide. It enhances collagen production by producing proline, which is a common amino acid involved in protein synthesis and a major component of collagen. The NS is also enriched with vitamins $\mathrm{E}, \mathrm{C}$ and zinc, which also promote pressure ulcer healing.
The presence of a wound may increase metabolic demands due to the metabolic response to injury. A direct correlation between malnutrition severity and the PUs has been reported. ${ }^{[28]}$ A number of studies have demonstrated that this relationship is bidirectional. ${ }^{[28-29]}$ Nutrition and hydration play an important role in preserving skin and tissue viability and in supporting tissue repair for PU healing. ${ }^{[30]}$ Apart from treating the subjects with off loading the bony prominences, care of the back \& pressure points, good hygiene, nutritional enrichment is equally important for healing of PUs. Our data suggests that the rate of PU healing is increased by good ulcer care and addressing the local factors which impair healing. At the same time, the nutritional indices are auto corrected as the healing progresses.

\section{Conclusion:}

In conclusion, malnutrition is a problem of high prevalence and impact in PU subjects of SCI. Given the high prevalence of malnutrition among subjects with PUs, performing a routine nutritional screening should result in early identification of residents with the risk of developing PUs. Further this article gives statistical evidence that healing of PUs is associated with an increase in $\mathrm{Hb}$ level and improving metabolic alterations. There seems to be a positive relationship between PU healing cascade and nutritional corrections.

\section{References}

1. Bhattacharya S, Mishra RK. Pressure ulcers: current understanding and newer modalities of treatment. Indian $\mathrm{J}$ Plast Surg 2015;48:4-16.

2. Ikechukwu EC, Ayodiipo IO, Emeka AD, Kayode AJ, Michael NI, Deborah OT. Prevalence and factors associated with healing outcomes of hospital-acquired pressure ulcers among patients with spinal cord injury. J Public Health Epidemiol 2012;4:44-7.

3. Krassioukov A, Eng JJ, Warburton DE, Teasell R. A systematic review of the management of orthostatic hypotension after spinal cord injury. Arch Phys Med Rehabil. 2009;90:876-885.

4. Johanne Alhaug, Caryl L Gay, Christine Henriksen, Anners Lerdal. Pressure ulcer is associated with malnutrition as assessed by Nutritional Risk Screening (NRS 2002) in a mixed hospital populationFood Nutr Res. 2017; 61(1): 1324230 .

5. Anderson K, Hamm RL. Factors that impair wound healing. J Am Coll Clin Wound Spec 2014;4:84-91.

6. Montalcini T, Moraca M, Ferro Y, Romeo S, Serra S, Raso MG, Rossi F, Sannita WG, Dolce G, Pujia A. Nutritional parameters predicting pressure ulcers and short-term mortality in patients with minimal conscious state as a result of traumatic and non-traumatic acquired brain injury. J Transl Med. 2015;13:305. 
7. MW Robinson, C Harmon, C O'Farrelly. Liver immunology and its role in inflammation and homeostasis. Cellular \& Molecular Immunology 13 (3), 267-276

8. Crispe IN. The liver as a lymphoid organ. Annu Rev Immunol 2009; 27: 147-163.

9. Nemeth E, Baird AW, O'Farrelly C. Microanatomy of the liver immune system. Semin Immunopathol 2009; 31: 333343.

10. O'Farrelly C, Crispe IN. Prometheus through the looking glass: reflections on the hepatic immune system. Immunol Today 1999; 20: 394-398.

11. Bunn FH. Anemia associata a malattie sistemiche croniche. In: Isselbacher KJ, Adams RD, Braunwald E, Petersdorf RG, Wilson JD (eds). 'Harrison's Principi di medicina interna' 1986, Vol. 2. Piccin: Padova, pp 2109-2112

12. Jarnum S, Lassen NA. Albumin and transferrin metabolism in infections and toxic diseases. Scand J Clin Lab Invest 1961; 13: 357-361.

13. Sehgal PB. Interleukin-6: a regulator of plasma protein gene expression in hepatic and non-hepatic tissues. Mol Biol Med 1990; 7: 117-125.

14. Andus T, Bauer J, Gerok W. Effects of cytokines on the liver. Hepatology 1991; 13: 364-368.

15. Expression of MMP-8 in Pressure Injuries in Spinal cord injury patients managed by negative pressure wound therapy or conventional wound care. Mukesh K Dwivedi, Amit K Bhagat, Rajeshwar N Srivastava, Amita Jain, Kavita Baghel, Saloni Raj. J Wound Ostomy Continence Nurs. 2017; 44(4):343-349.

16. Wians FR, Urban JE, Keffer JH, Kroft SH. Discriminating between iron deficiency anemia and anemia of chronic disease using traditional indices of iron status vs transferrin receptor concentration. Am J Clin Pathol 2001; 116: 446-447

17. Lenche Neloska1 *, Katerina Damevska2 , Andjelka Nikolchev1, Lidija Pavleska1, Biljana Petreska-Zovic1, Milenko Kostov. The Association between Malnutrition and Pressure Ulcers in Elderly in Long-Term Care Facility. Maced J Med Sci. 2016 Sep 15; 4(3):423-427.

18. James J. Optimal wound healing: a comprehensive approach through metabolic, anabolic and nutritional interventions.
Involuntary weight loss and its effect on the body to heal. WOUNDS. 2002;14(9):4-8.

19. Ortega-Andreu M, Pérez-Chrzanowska H, Figueredo R, Gómez-Barrena E. Blood loss control with two doses of tranexamic acid in a multimodal protocol for total knee arthroplasty. The open orthopaedics journal. 2011;5:44.

20. Schreml S, Szeimies R, Prantl L, Karrer S, Landthaler M, Babilas P. Oxygen in acute and chronic wound healing. British Journal of Dermatology. 2010;163(2):257-68.

21. Löndahl M, Fagher K, Katzman P. What is the role of hyperbaric oxygen in the management of diabetic foot disease? Current diabetes reports. 2011;11(4):285-93

22. Fuoco U1, Scivoletto G, Pace A, Vona VU, Castellano V. Anaemia and serum protein alteration in patients with pressure ulcers. Spinal Cord. 1997 Jan;35(1):58-60.

23. Perkash A, Brown M. Anemia in patients with traumatic spinal cord injury. J Am Paraplegia Soc 1986; 9: 10-152.

24. Corso F. Manuale di patologia clinica, 2nd edn. Masson: Milano, 1986, pp 75-88.

25. Turba RM, Lewis VI, Green D. Decubitus ulcer anemia: response to erythropoietin. Arch Phys Med Rehab 1992; 75: 498-500.

26. Waldman TA, Gordon RS, Rosse W. Studies on the metabolism of serum protein and lipids in patients with analbuminemia. Am J Med 1984; 37: 960-968

27. Mobarhan S. The role of albumin in nutritional support. J Am Coll Nutr 1988; 7: 445-452.

28. Park KH. A retrospective study using the pressure ulcer scale for healing (PUSH) tool to examine factors affecting stage II pressure ulcer healing in a Korean acute care hospital. Ostomy Wound Manage. 2014;60(9):40-51.

29. Fossum M, Ehnfors M, Svensson E, Hansen LM, Ehrenberg A. Effects of a computerized decision support system on care planning for pressure ulcers and malnutrition in nursing homes: an intervention study. Int $\mathrm{J}$ Med Inform. 2013;82(10):911-21.

30. Posthauer ME, Banks M, Dorner B, Schols JM. The role of nutrition for pressure ulcer management: national pressure ulcer advisory panel, European pressure ulcer advisory panel, and pan pacific pressure injury alliance white paper. Adv Skin Wound Care. 2015;28(4):175-88.

*Corresponding author:

Dr Rajeshwar Nath Srivastava, Professor, Dept. of Orthopaedic Surgery, King George's Medical University, Lucknow, U.P., India, Pin code-226003 Phone: +91 9415054330

Email: drrnsrivastava@yahoo.com

Financial or other Competing Interests: None. 